\title{
FRIGA, A New Approach To Identify Isotopes and Hyper-nuclei In N-Body Transport Models.
}

\author{
A. Le Fèvre ${ }^{1}$, J. Aichelin ${ }^{2,3}$, C. Hartnack ${ }^{2}$, Y. Leifels ${ }^{1}$ \\ ${ }^{1}$ GSI Helmholtzzentrum für Schwerionenforschung GmbH, Planckstr. 1, 64291 Darmstadt, Germany \\ 2 SUBATECH, IMT Atlantique, Université de Nantes, IN2P3/CNRS \\ 4 rue Alfred Kastler, 44307 Nantes cedex 3, France and \\ 3 Frankfurt Institute for Advanced Studies, Ruth Moufang Str. 1 \\ 60438 Frankfurt, Germany
}

(Dated: June 17, 2019)

\begin{abstract}
We present a new approach to identify fragments in computer simulations of relativistic heavy ion collisions. It is based on the simulated annealing technique and can be applied to n-body transport models like the Quantum Molecular Dynamics. This new approach is able to predict isotope yields as well as hyper-nucleus production. In order to illustrate its predicting power, we confront this new method with experimental data and show the sensitivity on the parameters which govern the cluster formation.
\end{abstract}

\section{INTRODUCTION}

In heavy ion reactions at energies between $20 \mathrm{MeV}$ and several GeV per nucleon, the formation of complex clusters is a key observable [1, 2], which is not understood in all details, yet. Sophisticated microscopic transport models [5 7] employing clusters as degrees of freedom have been developed, but they are not generally applicable to the collision energies under consideration or are constrained to small clusters $(\mathrm{A} \leq 3)$. Identifying clusters represents also a major challenge for transport models propagating only nucleons as relevant degrees of freedom. Omitting fragment formation makes the prediction of proton and neutron observables ambiguous because fragments have different kinematical properties than single nucleons.

Transport models based on the time evolution of the one-body density matrix, like BUU [3] or SMASH [4] cannot address this question directly without injecting sufficient phase space fluctuations into the system [8 10].

On their side, QMD approaches, which are based on Nbody theories [1] 13] propagate the correlations in time and, therefore, contain all necessary information to describe clusters.

Basic ways to identify clusters are to employ a coalescence model [17 20] or to use a minimum spanning tree (MST) procedure 21]. The first method needs various coalescence parameters for each isotope, is unable to deal with heavy clusters, and moreover a time has to be chosen at which the transport calculation is stopped and the coalescence procedure is applied. In a rapidly expanding system like at the end of a heavy ion collision the cluster yields depend crucially on this time. In addition, it has been shown for light clusters, that this time is different for different isotopes [14]. To study the production of light hypernuclei in the UrQMD model the coalescence model has recently been applied in refs. 15, 16. It turns out that there exists a choice of parameters for which the results are quite satisfying. The MST procedure - based only on proximity criteria in position and optionally momentum space - allows only for an identification of the fragments at the end of the reaction when the fragments and single nucleons have well separated from each other.

The drawback of both methods is that the study of the physical origin [21] of fragmentation is excluded. In addition, since the underlying transport models are semi-classical, binding energy modifications due to closed shells or pairing energies are neglected in these approaches.

\section{THE PRINCIPLES OF THE FRAGMENT RECOGNITION.}

Identifying fragments early, while the reaction is still going on, requires to define the most bound cluster partition out of a set of clusters pre-selected using momentum as well as coordinate space information, like in MST. This idea has been first introduced by Dorso et al. 22]. It has been further applied into the Simulated Annealing Clusterisation Algorithm (SACA) 23] in the late 1990's and has been successfully applied to understand experimental fragment charge distribution and spectra as well as bimodal distributions [21, 24, 25]. This procedure can be applied at different times during the collision to provide the time evolution of the most probable cluster distribution in terms of binding energy. It turns out that this method detects the final partitions early - that found by MST at very late times (typically $>200 \mathrm{fm} / \mathrm{c}$ ) -, right after the colliding system begins to separate, when the energetic collisions are over, as shown in [23]. By "final partition", we mean the asymptotic (late time) cluster distribution that would be detected by the same method within a transport model that would not introduce artificial time instabilities in the phase space distribution of nucleons. We want to stress at this point the fact that, when we are using for example a QMD transport model, we are dealing with a semi-classical approach, and not with a pure-quantum approach. This manifests itself on a long time scale when the fragments become unstable. The reason for this is that in these codes the ground state of the quantum hamiltonian is higher than the ground 
state of the classical hamiltonian. As a consequence, a semi-classical system can still emit particle when in the analog quantum system it is not possible anymore.

The seed, an ensemble of pre-clusters, for the annealing procedure is generated by using an MST. Then nucleons are exchanged between neighbouring fragments or single nucleons in all possible ways applying a simulated annealing technique, based on a Metropolis algorithm. Neglecting the interactions between nucleons of different clusters, but taking into account the interaction among the nucleons in the same fragment, this algorithm identifies the combination of fragments and free nucleons which has the most negative total binding energy - i.e. the most bound sum. The reason for this is the fact that fragments are not a random collection of nucleons at the end, but initial-final state correlations.

In SACA the nucleon-nucleon interactions taken into account to calculate the binding energies of clusters are a Skyrme potential supplemented by a Yukawa term QMD surface correction [1] - and a Coulomb potential. These potentials are also used for the propagation of the nucleons in the QMD transport model which was utilised for the time evolution of the reaction [11]. They are used for calculating a cluster binding energy in the following way: introducing (density dependent) two-body interactions among the nucleons which form a fragment, the internal fragment energy

$$
\begin{aligned}
& E_{B}=\langle H\rangle=\langle T\rangle+\langle V\rangle(N, Z) \\
&=\sum_{i} \frac{p_{i}^{2}}{2 m_{i}}+ \\
& \sum_{i} \sum_{j>i} \int f_{i}(\mathbf{r}, \mathbf{p}, t) V\left(\mathbf{r}, \mathbf{r}^{\prime}, \mathbf{p}, \mathbf{p}^{\prime}\right) f_{j}\left(\mathbf{r}^{\prime}, \mathbf{p}^{\prime}, t\right) \\
& \\
& \mathrm{d} \mathbf{r} \mathrm{d} \mathbf{r}^{\prime} \mathrm{d} \mathbf{p} \mathrm{d} \mathbf{p}^{\prime} .
\end{aligned}
$$

where $\mathbf{r}, \mathbf{p}$ is the particle phase-space position in the centre-of-mass of the collision, $m_{i}$ is its mass, $V$ is the potential, $f_{i}$ is the single-particle Wigner density

$$
f_{i}(\mathbf{r}, \mathbf{p}, t)=\frac{1}{\pi^{3} \hbar^{3}} \mathrm{e}^{-\frac{2}{L}\left(\mathbf{r}-\mathbf{r}_{\mathbf{i}}(t)\right)^{2}} \mathrm{e}^{-\frac{L}{2 \hbar^{2}}\left(\mathbf{p}-\mathbf{p}_{\mathbf{i}}(t)\right)^{2}}
$$

The potential $V$ consists out of a Skyrme type potential complemented by a Yukawa and a Coulomb potential. This combination of potentials we will denote as "basic". It has been shown in reference [11] that Eq. [1]reproduces very well the binding energies of nuclei with $A>5$ as given by the Bethe-Weizsäcker mass formula for ground state nuclei, $B_{B W, 0}$ (see Fig.12 of Ref. [11]). For nuclei with $A \leq 5$ this method provides slightly less bound values than the Bethe-Weizsäcker mass formula. This is taken into account in FRIGA by shifting accordingly the cluster ground state binding energy when calculating its excitation energy. Note that the nuclear densities in a cluster are here computed from the sole nucleons composing it, as if it were isolated, similarly to neglecting the nuclear force from external nucleons. The reason is that as soon as the asymptotic cluster partition is reached, the cluster binding energy must correspond to that of a free nucleus, therefore whose relevant density is that of its asymptotic state, i.e. close to the ground state. For this reason, the density used for calculating the cluster binding energy differs from that of the medium.

The SACA model has been extended in order to predict more realistically fragment yields in the isotopic degree of freedom, and to address hyper-nuclei production. For doing so, additional potentials (asymmetry energy and shell effects) enter the determination of the binding energy of primary clusters. In addition, when excited, those latter undergo a sequential secondary decay at very late times, when the long range Coulomb interaction between clusters becomes negligible (at the order of $1000 \mathrm{fm} / \mathrm{c}$ ). This new approach is dubbed FRIGA ("Fragment Recognition In General Application"). The basic idea of FRIGA is to use the same potentials (mean fields) as has been applied in the transport code and to add further interactions which are relevant for binding energies of nuclei, in particular shell effects.

\section{THE FEATURES OF FRIGA.}

In order to predict the isotope yields, we have extended the SACA cluster identification algorithm by including asymmetry energy, pairing and shell effects.

For the asymmetry energy we adopt the parametrisation from IQMD [12], the transport code which we use - in addition to BQMD - in the present article for the transport of the nucleons. The potential part of the asymmetry energy, which is repulsive, thus reads:

$$
B_{a s y}=E_{0}\left(\frac{\rho_{n}-\rho_{p}}{\rho_{B}}\right)^{2}\left(\frac{<\rho_{B}>}{\rho_{0}}\right)^{\gamma}
$$

where $E_{0}=23.3 \mathrm{MeV}$, and $\rho_{n}, \rho_{p}, \rho_{B}, \rho_{0}$ are the neutron, proton, baryonic and saturation densities, respectively. In the present work, by default, we take $\gamma=1$ (linear density dependence).

Note that the kinetic part of the asymmetry energy is carried by the nucleon momenta which, according to the Thomas-Fermi model, relates to the differences of the Fermi edges of neutrons and protons: For $\rho_{0}$ it corresponds to a value of about $9 \mathrm{MeV}$ in IQMD.

Another significant part of the binding energy of light isotopes are the shell structure and odd-even effects (pairing). In the conditions of high pressure and temperature where FRIGA is used to determine the pre-fragments, these structure effects are not well known. E. Khan et al. 33. showed that there are some indications that they can affect the primary fragments. The authors demonstrate that the pairing vanishes above a nuclear temperature $T_{V} \approx 0.5 \Delta_{\text {pairing }}$ (pairing energy). At the density of their fundamental state, the pairing energy tends to be negligible for heavy nuclei, with the pairing energy taken from the Bethe-Weizsäcker mass formula $\Delta_{\text {pairing }}=11.2 A^{-\frac{1}{2}} \mathrm{MeV}$ (positive for even-even and negative for odd-odd nuclei), whereas it is strong for light 
isotopes, like ${ }^{4} \mathrm{He}$ and ${ }^{3} \mathrm{He}$ with $12 \mathrm{MeV}$ and $6.9 \mathrm{MeV}$, respectively. In FRIGA, due to the minimisation of the binding energy, the primary fragments are expected to be produced quite cold on average, with $T \sim 1-2 \mathrm{MeV}$, and with a density close to that of their ground state, slightly below $\rho_{0}$ (typically between $\rho_{0} / 2$ and $\rho_{0}$ depending on the fragment size). Hence, their temperature could be below $T_{V}$ and one cannot neglect the pairing energy. The same might be true for shell effects which produce a visible enhancement of the measured fragment yields for closed shell nuclei. It will be a crucial point to determine whether these shell effects are already realised in the primary stage of the fragment production, or later due to secondary de-excitation.

In order to determine the contribution of all structure effects to the binding energy of primary clusters identified by FRIGA, we make two hypotheses, independent of the density and the average kinetic energy of the fragment environment.

First, the relative ratio of the nuclear structure contribution to the overall binding energy remains unchanged at moderate temperatures and at densities close to that of the fundamental state of the cluster.

Applying our first assumption that the ratio of the still "unknown" nuclear structure contribution to the binding energy $B_{\text {struct }}(Z, N, \rho, T)$ and the calculated binding energy $E_{B}(Z, N, \rho, T)$ of Eq. 1 is constant in the respective density and temperature ranges, one obtains

$$
\begin{aligned}
\frac{B_{\text {struct }}(Z, N, \rho, T)}{E_{B(\text { Sky }, Y \text { uk }, \text { Coul })}(Z, N, \rho, T)} & = \\
\frac{B_{\text {exp }, \text { struct }}(Z, N)}{B_{B W(\text { vol }, \text { surf }, \text { Coul })}(Z, N)} & =\text { const. }(Z, N)
\end{aligned}
$$

where $B_{B W(\text { vol,surf,Coul })}$ is the binding energy as given by the sum of the volume, surface, and Coulomb terms of the Bethe-Weizsäcker mass formula - considered as a ground state for $E_{B(S k y, Y u k, C o u l)}(Z, N, \rho=$ $\left.r h o_{0}, T=0\right)$, whereas $B_{\text {exp struct }}$ is the difference between the experimentally observed binding energy $B_{\text {exp }}$ and the prediction of the mass formula without pairing term, $B_{\text {exp struct }}(Z, N)=B_{\text {exp }}(Z, N)-$ $B_{B W(\text { vol,surf }, \text { Coul }, a s y)}(Z, N)$.

Our second hypothesis is that Eq. 1 remains the correct description of the binding energy if the nuclei are deformed or excited as it might happen for fragments identified by the FRIGA algorithm.

Under these assumptions we can express the nuclear structure contribution to the binding energy of a deformed cluster with $\mathrm{Z}$ protons and $\mathrm{N}$ neutrons in the following way:

$$
\begin{aligned}
B_{\text {struct }}(\rho, T, Z, N) & =\left(B_{\exp }(Z, N)-B_{B W n p}(Z, N)\right) \\
& \times \frac{E_{B}(\rho, T, Z, N)}{B_{B W n p, n a}(Z, N)}
\end{aligned}
$$

$E_{B}$ is the binding energy of Eq. 1 and $B_{B W n p, n a}$ is that given by the Bethe-Weizsäcker formula binding without asymmetry ("na") and pairing ("np") contributions. Isotopes and hyper nuclei which are not stable at all in nature, are discarded in FRIGA by assigning to them a very repulsive $E_{B}$. The complete total binding energy of a cluster with $\mathrm{N}$ and $\mathrm{Z}$, which is used in the annealing algorithm, will then be:

$$
B=E_{B}(Z, N)+B_{\text {asy }}+B_{\text {struct }} .
$$

in contradistinction to SACA in which only the first term is used.

The other new feature of FRIGA concerns the initial configuration of the SACA algorithm. There the simulated annealing procedure started out from the full cluster partition provided by the MST procedure, based on the distance of the nucleons in coordinate space. Subsequently SACA reorganises the partition in order to minimize the sum of the cluster binding energies. In FRIGA, "cold" MST primary clusters are removed from the ensemble, i.e.are kept as they are. The standard criterium for categorizing a fragment as "cold" is a maximum internal excitation energy of $1 \mathrm{~A} \mathrm{MeV}$ (see definition in Sect. (V). This new method is particularly meaningful for very peripheral collisions where the main spectator remnant (quasi-projectile or quasi-target) - well identified by the MST method - is at nearly zero excitation energy.

\section{EARLY FRAGMENT RECOGNITION.}

As already shown in [21], the application of the simulated annealing method to find the most bound configuration allows to identify fragments much earlier in the course of the heavy ion collision than MST. Fig. 1 illustrates this fact with the FRIGA clusterisation applied to BQMD [11] simulations of ${ }^{197} A u+{ }^{197} A u$ collisions at 600 $\mathrm{A} \mathrm{MeV}$ incident energy and an impact parameter of $4 \mathrm{fm}$. Fig. 1 top compares the time evolutions of the average multiplicity of light particles, $Z \leq 2$ of the projectile rapidity side obtained with MST and FRIGA. For the MST algorithm $\Delta p=0.6 \mathrm{GeV} / \mathrm{c}$ and $\Delta r=2.5 \mathrm{fm}$ have been utilized.

To compare to SACA directly (as published in Ref. 21]), we have only taken $E_{B}$ (Eq. (1) to calculate the binding energies in FRIGA and omitted the asymmetry energy $B_{a s y}$ and shell effects $B_{\text {struct }}$. However we have observed that the present results are not modified by the inclusion of these extra potentials. Obviously, similarly to what had been found in [21], the asymptotic values of the light fragment multiplicity is reached very early with FRIGA, at around $50 \mathrm{fm} / \mathrm{c}$, whereas MST needs at least $200 \mathrm{fm} / \mathrm{c}$ to obtain a stable configuration. The same fast convergence to the asymptotic value of the FRIGA results is seen in Fig. 10bottom for the observable $a_{2}$, which is relevant for the observation of bimodality in heavy ion collisions at low incident energies [25] and reflects the mechanical state of the system:

$$
a_{2}=\frac{Z_{1}-Z_{2}}{Z_{1}+Z_{2}}
$$




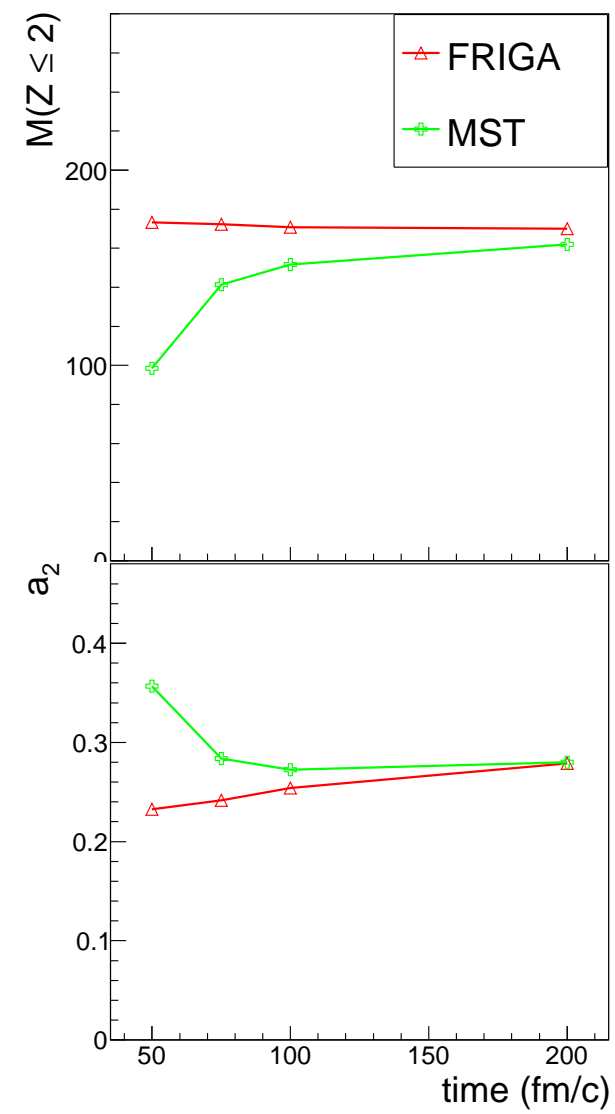

FIG. 1. BQMD (hard equation of state) predictions of ${ }^{197} A u+{ }^{197} A u$ collisions at $600 \mathrm{~A} \mathrm{MeV}$ incident energy for an impact parameter of $4 \mathrm{fm}$ (semi-central). Top: Event multiplicity of primary small fragments with charge $Z \leq 2$ identified on the projectile side as a function of time by FRIGA and MST (respectively red triangles and green crosses). Bottom: as top panel for the relative asymmetry between the primary two largest charges of fragments identified on the projectile side (see text for the definition of $a_{2}$ ).

where $Z_{1}$ is the largest and $Z_{2}$ the second largest charge observed on the projectile (or target) side at the end of the collision. In general, we observe for spectator fragmentation in heavy ion collisions with an incident energies around or below $1 \mathrm{~A} \mathrm{GeV}$, that FRIGA can identify final fragments as early as twice the passing time of projectile and target, i.e. the time that they would need to completely cross each other at zero impact parameter if the nuclei were fully transparent.

\section{DE-EXCITATION OF EXCITED FRAGMENTS}

Most of the clusters identified with FRIGA are relatively cold with a value of their binding energies close to the the ground state energy. They can either be overbound (negative binding energy below that of the ground

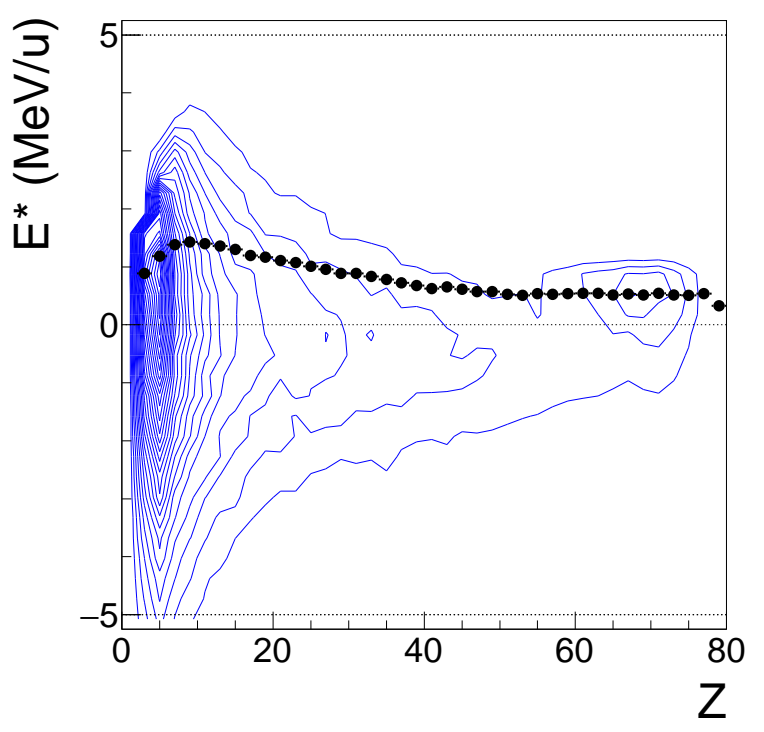

FIG. 2. Predictions of the BQMD transport code (hard equation of state) for ${ }^{197} A u+{ }^{197} A u$ collisions at $600 \mathrm{~A} \mathrm{MeV}$ incident energy with impact parameters below $12 \mathrm{fm}$. A minimum bias distribution was generated. The figure demonstrates the resulting excitation energies of the primary clusters identified by FRIGA as a function of their charge. The identification of clusters was performed between 50 and $75 \mathrm{fm} / \mathrm{c}$. This is two and three times longer than needed by the colliding system to separate. The contour plot represents the double differential probability distribution (linear scale). The black filled circles display the average values of positive excitation energies.

state) - and we keep them as they are - or slightly excited. These pre-fragments, called also "primary" fragments, can be produced "non relaxed" in shape and density, i.e. deformed with respect to their fundamental state. Most of the excitation energy is due to the difference in surface energy between the reconstructed state and the respective ground state of the nucleus.

Fig. 2 illustrates this variety of excitation energies of primary clusters as a function of their charge (blue contour lines), as identified by FRIGA after BQMD simulations of minimum bias ${ }^{197} A u+{ }^{197} A u$ collisions at $600 \mathrm{~A}$ $\mathrm{MeV}$. In FRIGA, we define the excitation energy $E^{*}$ of a fragment by

$$
E^{*}=\left(E_{B}+B_{a s y}\right)-B_{B W n p}(Z, N) .
$$

Here, shell effects are not taken into account, since they contribute little to the excitation energy and the BetheWeizsäcker formula includes them only in parts (pairing energy). In cases where the asymmetry energy is not taken into account when calculating the binding energy, it is neither computed in the ground state binding energy that is here the Bethe-Weizsäcker formula. We take the cold liquid-drop (at normal density) formula as if it describes the "natural" fundamental state of a cold nucleus as constructed by the QMD transport model (compare 


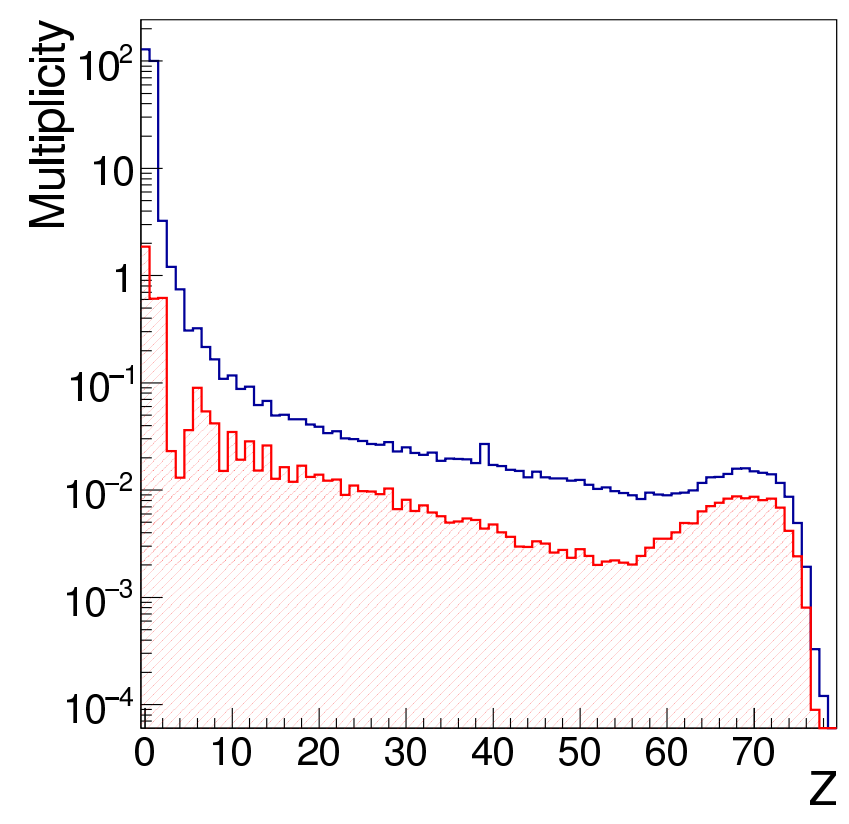

FIG. 3. The results for BQMD-FRIGA simulations of the average fragment multiplicity as function of their charge $Z$ are shown for the same reaction as Fig. 2 The blue full line and the red hashed area show the overall (secondary and cold primary) fragment multiplicity and that of fragments resulting from secondary decays, respectively. In the cluster recognition procedure with FRIGA neither asymmetry energy nor pairing or structure effects have been taken into account.

Fig. 12 of [11]). We have obtained quantitatively similar results for excitation energies of primary fragments at incident energies between $50 \mathrm{~A} \mathrm{MeV}$ and $1 \mathrm{~A} \mathrm{GeV}$. Positive values of the excitation energy correspond to hot primary clusters. Negative values represent nuclei which are over-bound. Over-binding may occur in a semi-classical approach because the ground state of the nucleus may not correspond to the lowest energy state of the quantal Hamiltonian. Hence, we assume that over-bound clusters are in their fundamental state with zero excitation energy. The black points in Fig. 2 represent the average value of positive excitation energies - zero excluded - as a function of the fragment charge. We observe that it hardly exceeds $1 \mathrm{~A} \mathrm{MeV}$. This means that the primary fragments produced by FRIGA are quite cold. This is also true for lower incident energies of around $100 \mathrm{~A} \mathrm{MeV}$. Those primary fragments which have an excitation energy exceeding $1 \mathrm{~A} \mathrm{MeV}$ can be considered as "hot" and should undergo a secondary decay. Since the highest excitation energies remain quite low, typical de-excitation is done via sequential evaporation or fission. For simulating this process, we use the GEMINI++ code [26] the most recent $\mathrm{C}++$ version of GEMINI - which evaluates the production cross sections for secondary reaction products after possible particle evaporation and/or fission.
Fig. 3 compares the overall (secondary and cold primary nuclei) charge yield (blue line) obtained at twice the passing time in minimum bias BQMD-FRIGA simulations of ${ }^{197} A u+{ }^{197} A u$ collisions at $600 \mathrm{~A} \mathrm{MeV}$ incident energy with the yield of fragments created by secondary decays (red-hashed area). We observe that the contribution of secondary decays become non-negligible for projectile/target remnants and for $\alpha$ particles: here secondaries contribute up to $50 \%$ to the total $\alpha$ yield. In addition, we observe that the secondary distribution exhibits an odd-event $\mathrm{Z}$ staggering at small $\mathrm{Z}$ which results from the pairing effects included in GEMINI++.

\section{BENCHMARKING IN THE SPECTATOR FRAGMENTATION REGIME.}

One of the main features of the spectator fragmentation at relativistic incident energy has been discovered by the ALADiN Collaboration in the late 1990's, dubbed "Rise and Fall" [1] curve, exhibiting a universal behaviour which is essentially independent of the beam energy and scales with the system size. This curve represents the average multiplicity of intermediate mass clusters $(2 \leq Z \leq 30)$ as a function of $Z_{\text {bound }}$ (total charge bound in fragments with $Z \geq 2$, which scales to the centrality of the collision).

It has been shown that the SACA approach, using BQMD as program for the time evolution of the nucleons, can reproduce this curve [21]. Similar analyses applying MST do not give an equally good description [28], illustrating that the "Rise and Fall" is a very sensitive and challenging observable for clustering methods.

However, as far as charge distributions are concerned, this lower accuracy of MST is less visible, especially in central collisions. It has been shown in [27], that MST used at late times on BQMD simulated collisions provides a fair agreement with experimental charge distributions.

To see whether FRIGA - including secondary decays - reproduces the SACA results on the ALADiN "Rise and Fall", we applied the FRIGA algorithm to minimum bias ${ }^{197} A u+{ }^{197} A u$ reactions at $600 \mathrm{~A} \mathrm{MeV}$, using only $E_{B}$ as for the calculation of binding energies. The result is shown in Fig. 4. There we display the average multiplicity of intermediate mass fragments $(2<Z \leq 30)$ on the spectator side (top) and the charge of the largest fragment detected on the spectator side as a function of $Z_{\text {bound }}$. The model predictions are compared with the most recent ALADiN data [S254 experiment, courtesy of the ALADiN2000 collaboration], obtained with an upgraded set-up detailed in 30] and 29]. Here again, MST partitions, obtained at $200 \mathrm{fm} / \mathrm{c}$, fail to reproduce the experimental findings. On the contrary, the FRIGA approach - as soon as twice the passing time - predicts those data with good agreement. We observed that the secondary decays do not modify sensitively the results in these representations. The results of the GEMINI calculations are shown as dashed lines in Fig. 4 


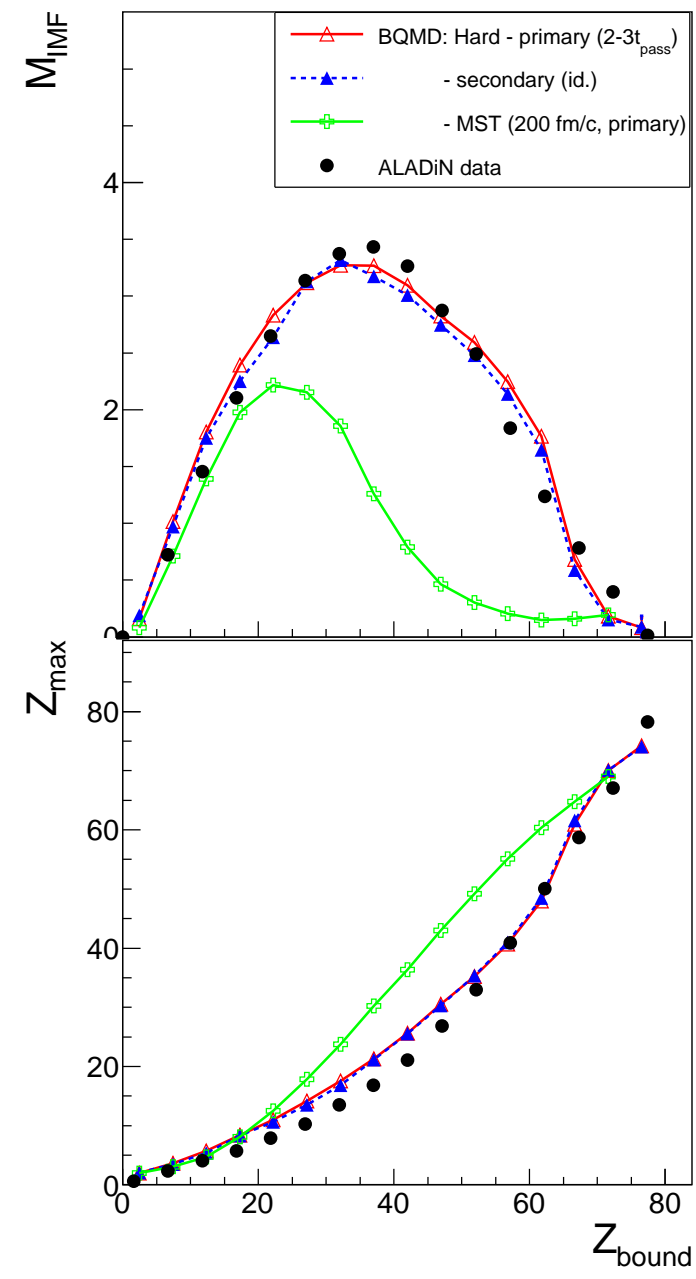

FIG. 4. BQMD (hard equation of state) predictions of ${ }^{197} A u+{ }^{197} A u$ collisions at $600 \mathrm{~A} \mathrm{MeV}$ incident energy for impact parameters $\mathrm{b}=0-12 \mathrm{fm}$, compared with the ALADiN S254 (2000) experimental data. Top: "Rise and Fall" curve (average multiplicity of IMF's as a function of the total charge of complex fragments with $Z \geq 2$, on the projectile side, dubbed $Z_{\text {bound }}$. The green crosses depict the BQMD cluster partitions identified with the MST method at $200 \mathrm{fm} / \mathrm{c}$ (primary clusters). The blue full and red open triangles show respectively the primary and secondary cluster partitions identified by FRIGA at 2 and 3 times the passing time (predictions of both times averaged to decrease the statistical fluctuations). Bottom: in the representation of the average largest $\mathrm{Z}$ as a function of $Z_{\text {bound }}$.

In order to estimate how much the equation of state (EoS) adopted in the transport model influences the results, we compare in Fig. 5] the experimental data with the BQMD-FRIGA predictions using three different equations of state: hard ( $\mathrm{H}$, red open triangles), soft (S, blue full triangles), soft with momentum dependent interaction (green crosses). The parameters adopted for the BQMD Skyrme potential

$$
U(\rho)=\alpha \frac{\rho}{\rho_{0}}+\beta{\frac{\rho}{\rho_{0}}}^{\gamma}+\delta \log ^{2}\left(\varepsilon(\Delta \mathbf{p})^{2}+1\right) \frac{\rho}{\rho_{0}}
$$

\begin{tabular}{lccccc}
$\mathrm{EoS}$ & $\mathrm{K}(\mathrm{MeV})$ & $\alpha(\mathrm{MeV})$ & $\beta(\mathrm{MeV})$ & $\gamma$ & $\delta(\mathrm{MeV})$ \\
\hline $\mathrm{H}$ & 380 & -124 & 70.5 & 2. & 0 \\
$\mathrm{~S}$ & 200 & -356 & 303 & $7 / 6$ & 0 \\
$\mathrm{SM}$ & 200 & -390.1 & 320.3 & 1.14 & 1.57
\end{tabular}

TABLE I. Parameter sets for the nuclear equation of state used in the BQMD model. $\mathrm{K}$ is the nuclear incompressibility modulus derived from the curvature of the potential at $\rho=\rho_{0}$

in the three configurations are listed in Table \.

Like the hard $(\mathrm{H})$ EoS, the soft EoS with momentum dependent interaction (SM) reproduces well the experiment when fragments are identified with FRIGA at around twice the passing time. BQMD with momentum dependent interactions (m.d.i.) is not stable asymptotically: with SM, the "Rise and Fall" results change noticeably with time. Therefore we do not pursue this approach further in this paper. Adopting a soft EoS without m.d.i. (S) does not reproduce the experimental data.

\section{ASYMMETRY ENERGY, SHELL EFFECTS AND SECONDARY DECAYS}

In order to illustrate the influence of the various new components of the binding energy of clusters in FRIGA, we compare the charge and light isotope yields of primary fragments exhibited by different compositions of the cluster binding energy in Fig. 6. In order to infer the isotopic dependence, we have used predictions of the IQMD code [12] which, unlike BQMD, explicitly treats neutrons and protons with respect to mean field and collisions and includes in its dynamics the proton-neutron asymmetry potential $B_{a s y}$. For benchmarking we select central ${ }^{129} \mathrm{Xe}+{ }^{124} \mathrm{Sn}$ collisions at $100 \mathrm{~A} \mathrm{MeV}$ incident energy for two reasons: first, they have been measured and isotopically resolved by the INDRA detector (see below), second, they allow to probe the binding energy configurations over a large variety of isotopes, in a strongly dynamical environment. Here, four different approaches are compared: MST alone (at $200 \mathrm{fm} / \mathrm{c}$, based on the coordinate space proximity of nucleons), minimization with FRIGA employing $E_{B}$ only, with $E_{B}+B_{a s y}$, with $E_{B}+B_{a s y}+B_{\text {struct }}$, respectively. Like in the following we consider the cluster partitions identified at the earliest possible time when they have reached their asymptotic characteristics. This time depends on the cluster recognition method used: It is typically twice the passing time with FRIGA, and $200 \mathrm{fm} / \mathrm{c}$ with MST. As already quoted, provided the transport model does not induce artificial modifications of the phase space extension of nucleons at late times, and the primary fragments are not excited, (early) FRIGA and (late) MST cluster distribution should be quite identical. But since both conditions are not perfectly fulfilled with the QMD transport model, the both approaches differ a bit. From the $\mathrm{Z}$ yields of primary fragments (Fig. 6 top), we observe first that the MST predictions do not differ strongly from 


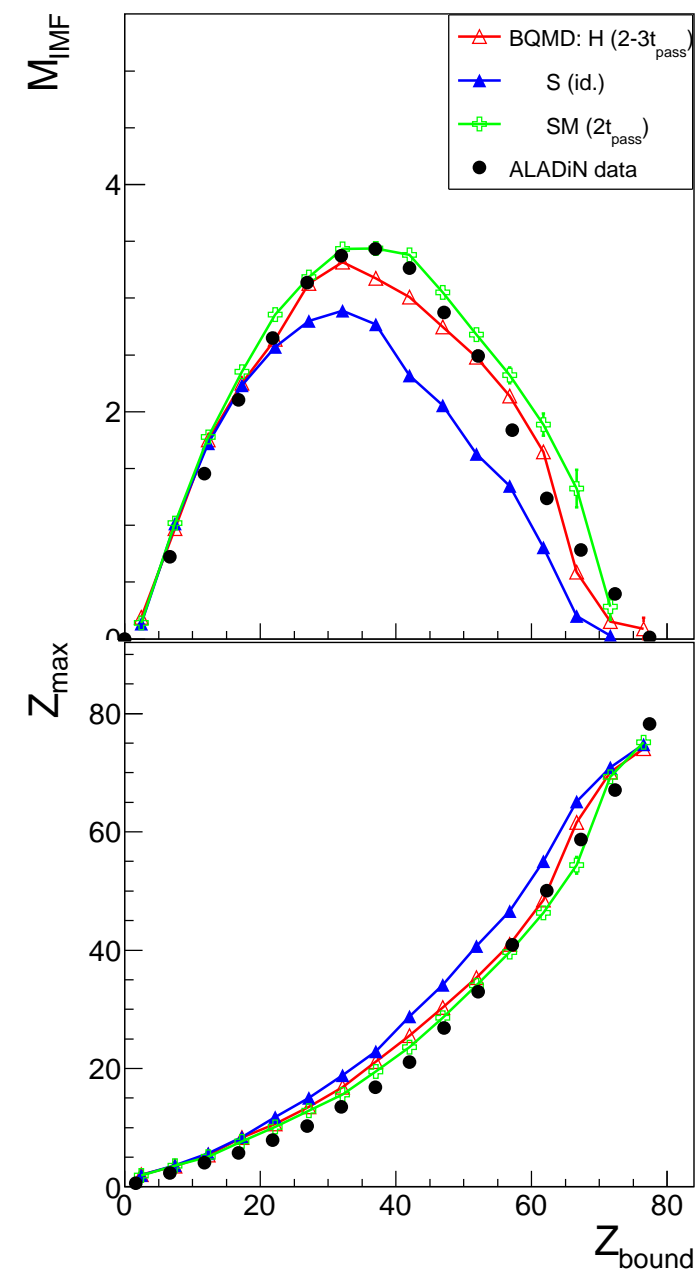

FIG. 5. Same as Fig 4 the FRIGA cluster partitions, after secondary decays, are compared to ALADiN experimental data. Three different parameterisations for the nuclear EoS in BQMD: hard $(\mathrm{H})$, soft $(\mathrm{S})$, and soft with momentum dependant interaction (SM), depicted respectively by the red open triangles, the blue full triangles and the green open crosses. The time interval adopted is $2-3$ times the passing time at this energy.

those of FRIGA, apart for large clusters whose yields are under-predicted by MST. Second, there is no strong influence of the various FRIGA approaches: the effects of the asymmetry and of the shell structure on the $\mathrm{Z}$ yield are very small. A stronger influence is visible if one studies the mass distribution of small isotopes, depicted in Fig. 6 bottom. We observe that MST exhibits broader distributions, because it is not constrained by the vetoing of unphysical isotopes - like ${ }^{8} \mathrm{Be}$ - and is based only on the phase space proximity. The asymmetry potential (here with a linear density dependence) tends by nature to narrow the distributions around $\mathrm{N}=\mathrm{Z}$. Shell effects would enhance or reduce the yields of particular isotopes, tempting to restore the natural abundances, according to the deviation of the experimental mass of the one given by the liquid drop model. For instance, $\alpha$ particles in this respect are highly favored because of their strong pairing energy.

In Fig. 7 the results of the FRIGA approach are compared to experimental data that have been measured by the INDRA detector at GSI Darmstadt [34]. The centrality of the events has been selected by means of the total transverse energy $E_{12}^{\perp}$ of detected nuclei with charge $\mathrm{Z}=1$ and 2 , similarly to Ref. [34]. The $10 \%$ most central collisions correspond to $E_{12}^{\perp}>1440 \mathrm{MeV}$. In order to enhance the reliability of the experimental yields, we have selected events where at least $70 \%$ of the total charge has been detected. To be able to compare the model predictions to the experimental data, we have filtered the IQMD-FRIGA events by a software replica of the INDRA acceptance. Fig. 7 top shows that the charge yield of the FRIGA primary clusters, detected in IQMD events, is close to the experimental data over the broad range of charges whereas the simple phase space proximity criteria used by MST does not give the correct slope. FRIGA predicts too many hydrogens, due to its lower efficiency in detecting helium fragments in the hot environment of central collisions. This may indicate that a more complex mechanism rules the production of the lightest isotopes in a hot expanding environment, as pointed out in [2]. Considering the isotope yields of Fig. 7 bottom, primary isotopes, given by FRIGA, reproduce fairly well the experimental yield starting from $A=6$.

Up to now we have not included secondary decays. To illustrate their effects on fragment yields, we choose the FRIGA strategy including the asymmetry energy and shell effects. Similar results are observed when considering only the basic potential for the binding energy. The results are shown as black lines in Fig. 8 for secondary fragments partitions, compared with the primary yield (red lines). The main consequence of secondary deexcitations is an increase of the yield of small fragments (with $Z<5$ ) at the cost of larger ones, which brings to a better agreement with the INDRA experimental charge distribution. The main channels of de-excitations are the emissions of neutrons and alphas particles, which is reflected by the enhancement of this latter ones as seen in Fig. 8 bottom. From the isotope yields, we conclude that shell effects in primary clusters are too weak to exhibit a clear difference. The reproduction of experimental yields of alpha particles is slightly improved by shell effects (after secondary decays) on a way, but on the other one, it is worsened for ${ }^{9} \mathrm{Be}$. The absence or not of primary shell effects is not obvious, because, in order to be stable, even primary fragments must be quite cold - therefore shell effects may still survive. On the other side, they are not in vacuum but tightly surrounded by a hot medium whose temperature is of the order of $6 \mathrm{MeV}$ in these collisions, as shown in 34. This may prevent a realization of structure effects inside the clusters in an early phase.

As we have seen when inspecting the width of the isotope mass distributions, the asymmetry energy in primary clusters is a key ingredient of the binding energy 

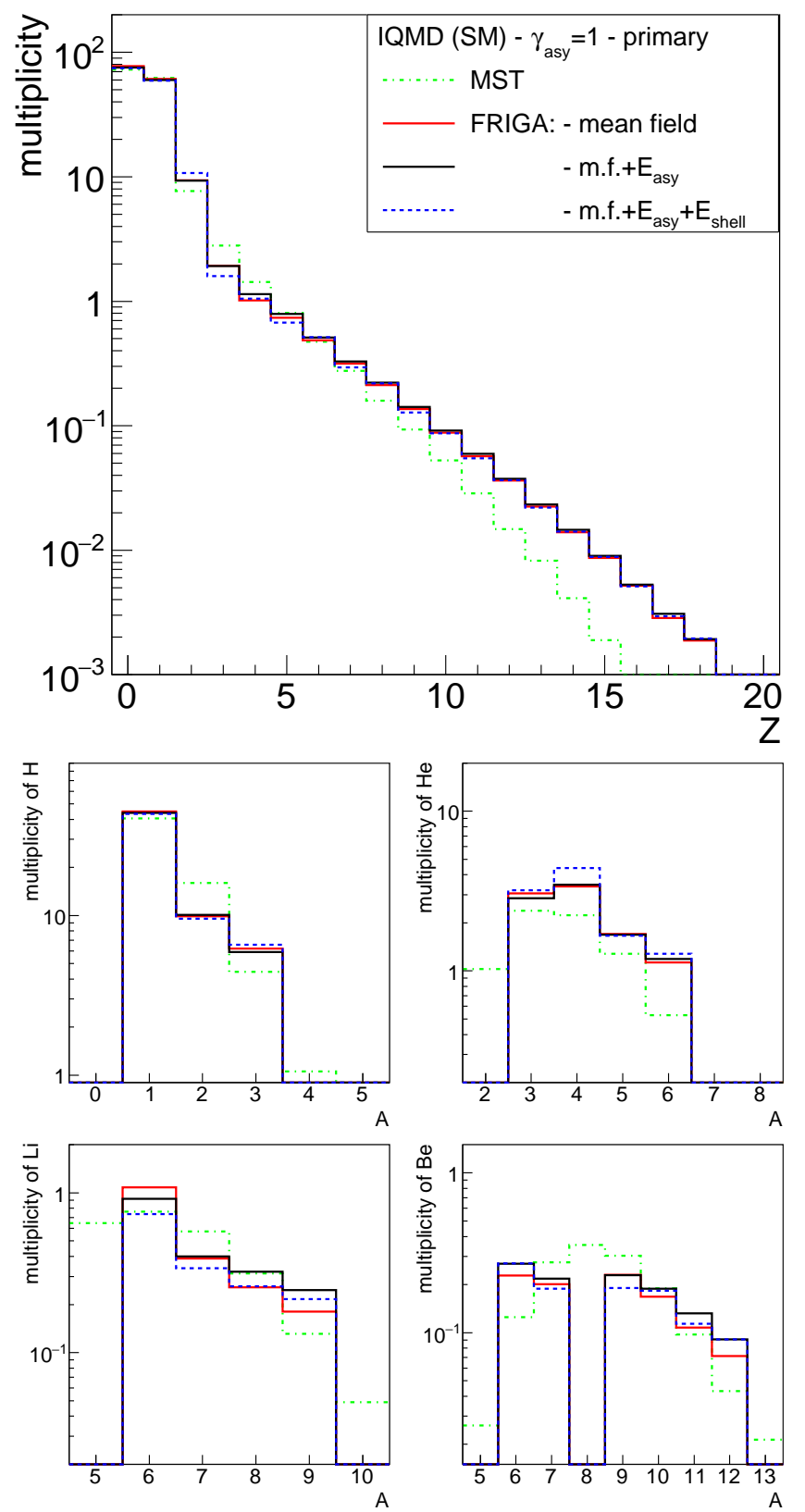

FIG. 6. IQMD (SM EoS) predictions of ${ }^{129} \mathrm{Xe}+{ }^{124} \mathrm{Sn}$ collisions at $100 \mathrm{~A} \mathrm{MeV}$ incident energy for an impact parameter $b<2.8 \mathrm{fm}$ ( $10 \%$ most central collisions) and an exponent of the asymmetry potential $\gamma_{a s y}=1$. Top panel: Average yields in an event of primary clusters as a function of their charge. Bottom four-panels: mass distributions of primary isotopes of hydrogen (top left), helium (top right), lithium (bottom left) and beryllium (bottom right). Three different FRIGA strategies are shown (applied at twice the passing time): with the basic potential only (red full lines), with including asymmetry potential (black full lines), and adding the shell energy (blue dashed lines). They are compared to the result of the minimum spanning tree method (green dotted dashed line). Colors in online version.
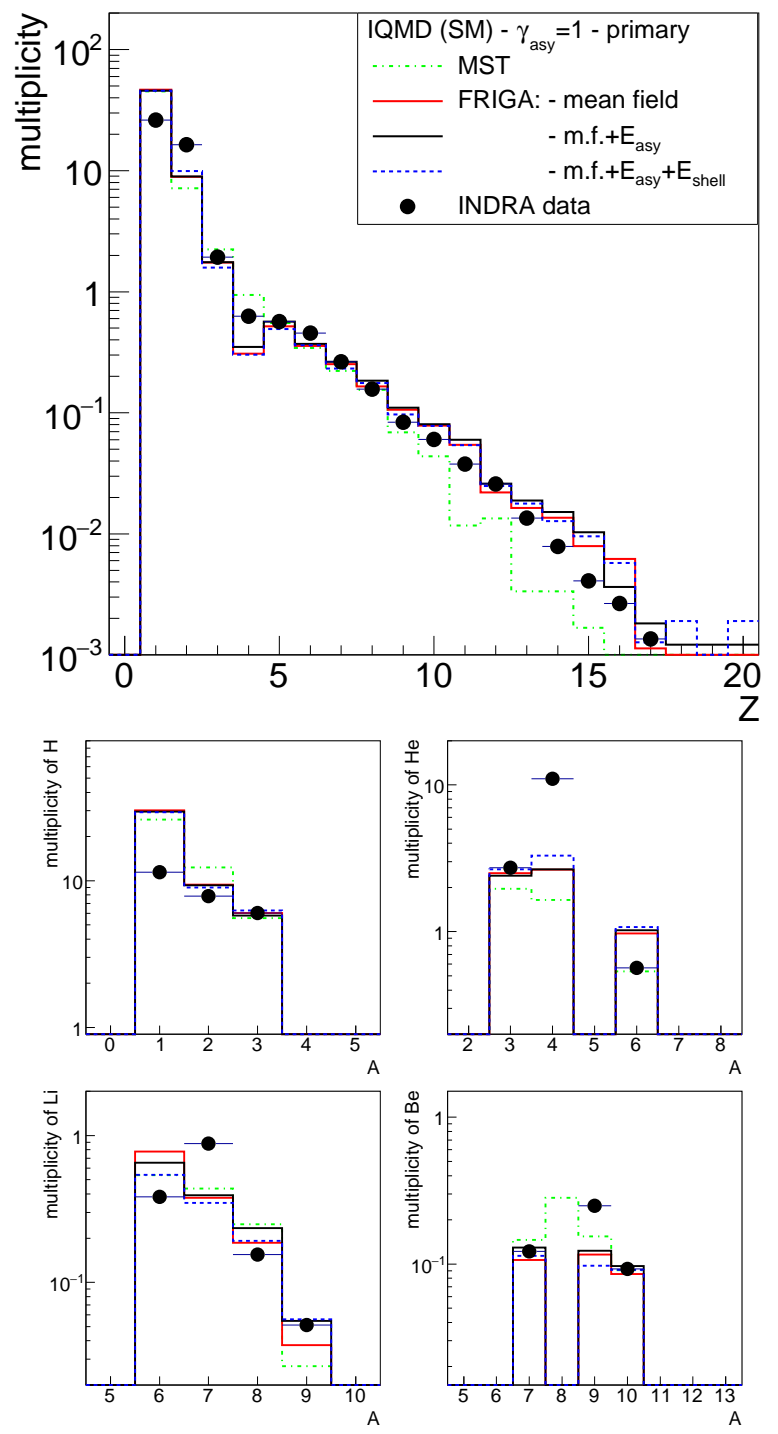

FIG. 7. Same as Fig. 6 compared with the INDRA experimental data (points). Predictions are filtered with the software replica of the INDRA acceptance.

for describing correctly the final isotope distributions. Therefore, it may be possible that the stiffness of its density dependence has a measurable influence on the observables. Fig. 9 displays the evolution of $B_{a s y}$ as a function of the density for various values of the exponent $\gamma_{\text {asy }}$. A larger exponent implies a stronger asymmetry potential at supra-saturation densities, and reversely at sub-saturation densities. The cluster internal density that we call "intrinsic" - is determined in the very same way as in IQMD [12]:

$$
\rho_{\text {int }}^{i}\left(\vec{r}_{i}\right)=\frac{1}{(\pi L)^{3 / 2}} \sum_{j \neq i} \mathrm{e}^{-\left(\overrightarrow{r_{i}}-\overrightarrow{r_{j}}\right)^{2} / L}
$$

As already quoted, the density used for calculating the binding energy in FRIGA is not that of the medium, but 

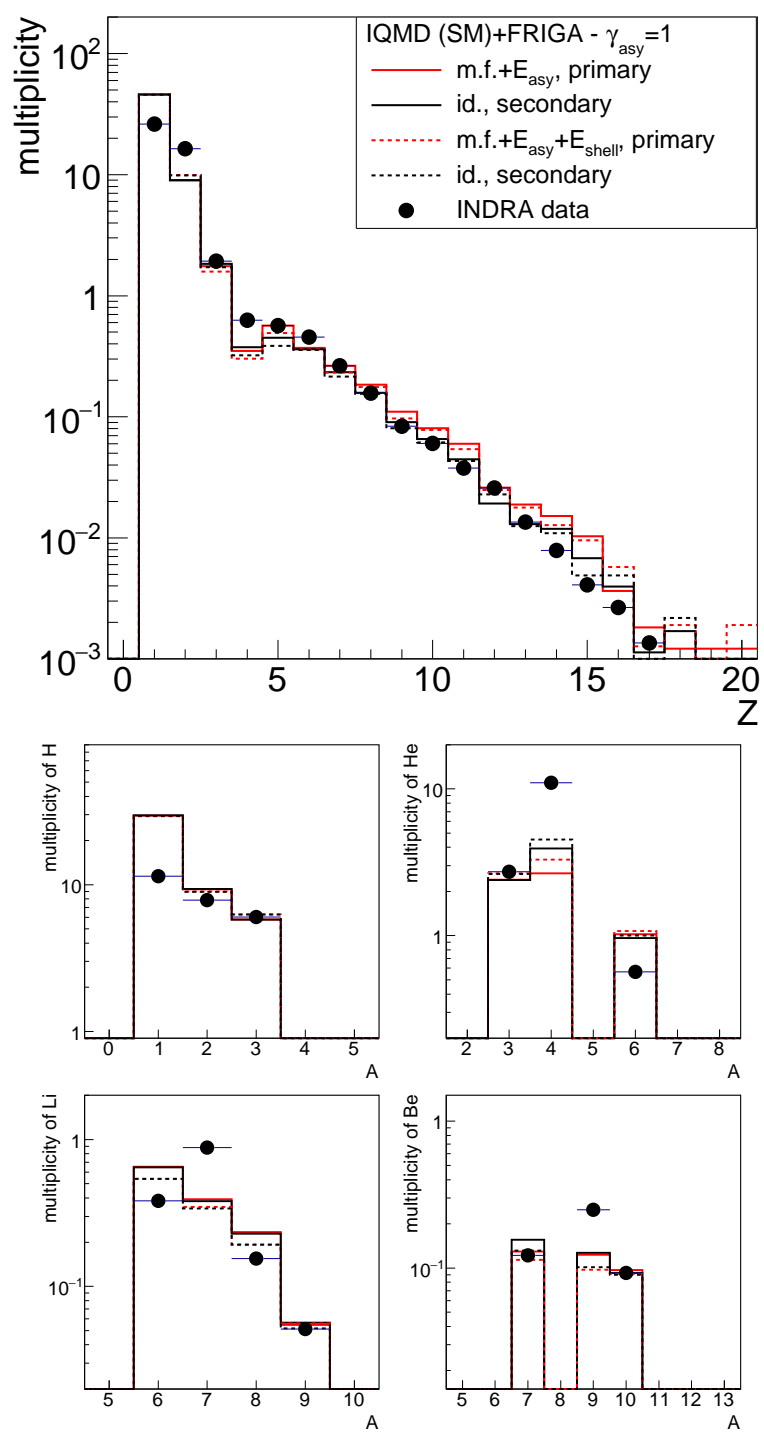

FIG. 8. Same as Fig. 7 comparing the INDRA experimental data with FRIGA predictions before (red lines) and after (black lines) secondary decays, with the FRIGA strategy including asymmetry energy (with $\gamma_{a s y}=1$ ).

that intrinsic to the cluster which is typically close to that of its ground state. Therefore, the cluster formation in FRIGA probes only sub-saturation densities typically ranging between 0.3 and 0.8 times $\rho_{0}$, as illustrated in Fig. 10 for the case of ${ }^{129} \mathrm{Xe}+{ }^{124} \mathrm{Sn}$ central collisions at $100 \mathrm{~A} \mathrm{MeV}$ incident energy. The average density increases with the fragment size. Hence, the strongest sensitivity on the stiffness of the asymmetry energy is expected for small to intermediate mass fragments, i.e. for $A \leq 20$. On its side, the average density of the medium has been observed to be close to $\rho_{0}$ when the partition of clusters identified by FRIGA are first stabilized. The fragments identified by FRIGA have a smaller density, typically around $\rho=\rho_{0} / 2$ for intermediate mass fragments, and

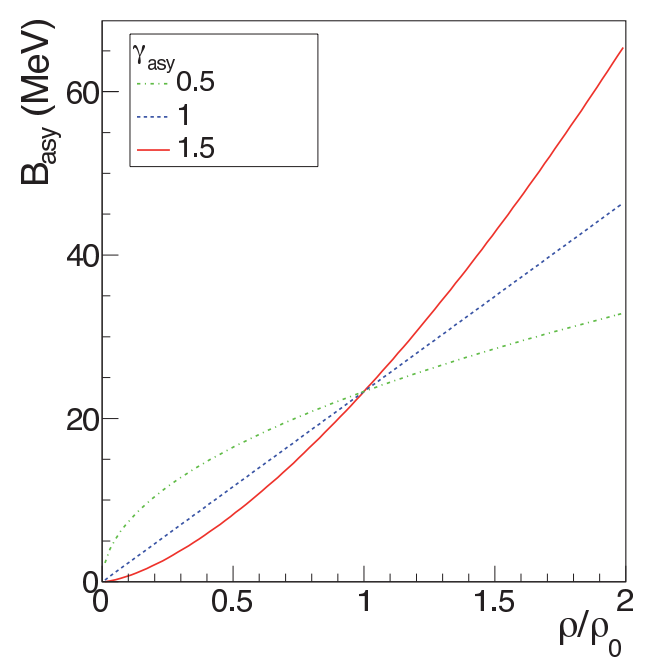

FIG. 9. Density dependence of the potential part of the asymmetry energy as used in FRIGA and IQMD for various values of the exponent $\gamma_{a s y}: 0.5$ (soft equation), 1 (stiff) and 1.5 (super-stiff), respectively displayed by green dasheddotted, blue dashed and red full lines.

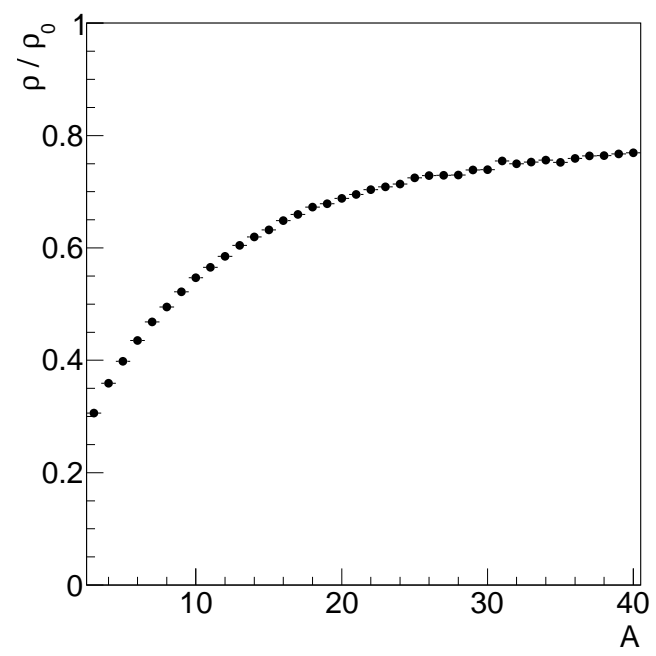

FIG. 10. Average values of the internal density of clusters scaled to the saturation density, as a function the mass number of primary clusters identified by FRIGA (with only the basic potential in the binding energy) at twice the passing time, out of IQMD (SM EoS, $\left.\gamma_{a s y}=1\right){ }^{129} \mathrm{Xe}+{ }^{124} \mathrm{Sn}$ central collisions at $100 \mathrm{~A} \mathrm{MeV}$ incident energy.

around $\rho=\rho_{0} / 5$ for the light $Z<3$ isotopes. In particular during the maximum overlap of the colliding system, nearby nucleons can happen to form a dense group, but they have quite different velocities in the beam direction. They do, however, not form a common fragment because its internal kinetic energy would be too high.

For the same system we show in Fig. 11 the sensitivity of fragment partitions on $\gamma_{a s y}$, as predicted by 

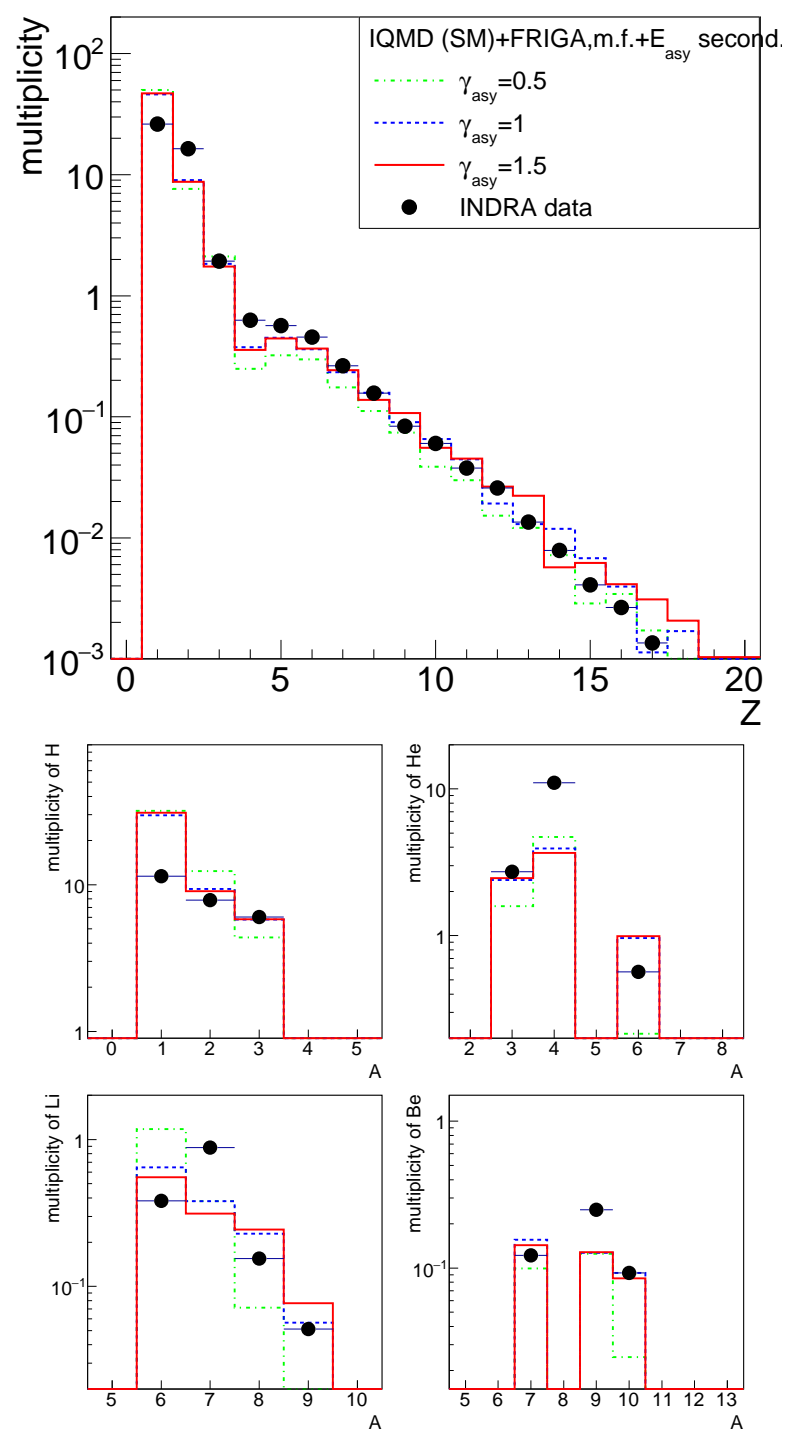

FIG. 11. Same as Fig. 7 comparing the INDRA experimental data with FRIGA predictions after secondary decay with asymmetry potential included, for the three values of the power exponent $\gamma_{a s y}$ of $E_{a s y}$ shown in Fig. 9 with corresponding line styles.

FRIGA. We use the basic and asymmetry potentials to calculate the cluster binding energy and observe that there is no strong influence on the charge multiplicities (Fig. 11t top), except for beryllium as seen in Fig. 11 bottom right. The reason is that a stronger asymmetry energy (therefore lower $\gamma_{a s y}$ at sub-saturation density) disfavours beryllium isotopes other than ${ }^{8} \mathrm{Be}$, but this latter decays into two alpha particles in the final secondary deexcitation procedure. The mass distributions of light isotopes (Fig.11bottom) are strongly influenced by $\gamma_{a s y}$, in particular the heaviest elements. Concerning hydrogen, deuterons and tritons, their yields show a slight sensitivity to $\gamma_{a s y}$. The general trend is that the softer the asymmetry potential (smaller $\gamma_{a s y}$ ), the narrower is the mass distribution around $A=2 Z$. Here, comparing our results with the experimental distributions of beryllium and lithium, we observe that a moderately soft asymmetry potential is favoured.

\section{THE HYPER-NUCLEUS FORMATION.}

A hyper-nucleus is a nucleus which contains at least one hyperon $(\Lambda(u d s), \ldots)$ in addition to nucleons. Here we will restrict ourselves to hyper-nuclei composed by $\Lambda_{0}$ hyperons. Extending FRIGA to the strange sector requires the knowledge of the hyperon- $\mathrm{N}($ here $\Lambda \mathrm{N})$ potential. In this first study, we consider the strange quark as inert and use $V_{\Lambda N}=\frac{2}{3} V_{N N}$ for protons as well as for neutrons. Similarly, we consider the case of multiple strange nuclei as well, in which more than one hyperon is bound in a fragment. There, the coupling of $2 \Lambda^{\prime} s$ contributes with the potential $V_{\Lambda \Lambda}=\left(\frac{2}{3}\right)^{2} V_{n N}$. In the present approach we neglect a possible contribution of the hyperons to the asymmetry energy, and take, as far as the asymmetry energy is concerned, only the contribution of the core of non-strange nucleons as if it were decoupled from the hyperon. Since for hyper-nuclei the pairing and shell contributions to the binding energy are not well known, we neglect the $B_{\text {struct }}$ contribution.

Using these modifications of the potentials, FRIGA identifies hyper-nuclei with the same procedure as non strange fragments. In the underlying transport program, $\Lambda$ 's are produced in different reactions: $\bar{K}+N \rightarrow \Lambda+\pi$, $\pi+N \rightarrow \Lambda+K^{+/ 0}, \pi^{-}+p \rightarrow \Lambda+K_{0}, p+p \rightarrow \Lambda+X$. Their abundance, position and momentum distributions are strongly influenced by the reaction kinematics, the nuclear equation of state and the in-medium properties of the $K^{+}$and $K^{-}$(kaon potential, etc.) [35].

Hyper-nuclei are produced when a cluster in coordinate and momentum space absorbs a hyperon. In heavyion collisions at relativistic energies, the hyperon distribution is strongly peaked around mid-rapidity whereas the large fragments have rapidities close to the beam or target rapidity. The closer the rapidity of the hyperon approaches - by production or by subsequent collisions - the target/beam rapidity, the larger is the probability that it can be absorbed by one of the heaviest fragments. Heavy hyper-nuclei are therefore produced not far away from beam/target rapidity. Hyperons can also form light clusters at mid-rapidity with other nucleons. There, the probability decreases with the cluster size because it is increasingly difficult to form large clusters out of a gas of nucleons. Whereas the large clusters in the beam/target rapidity regime can be identified quite early, the light clusters at mid-rapidty are formed late and many of them dissolve due to the interactions with the surrounding nucleons which form a gas at high temperature as compared to the cluster binding energy.

As discussed in the previous chapters, the ingredients of the cluster binding energy influence the light isotope 


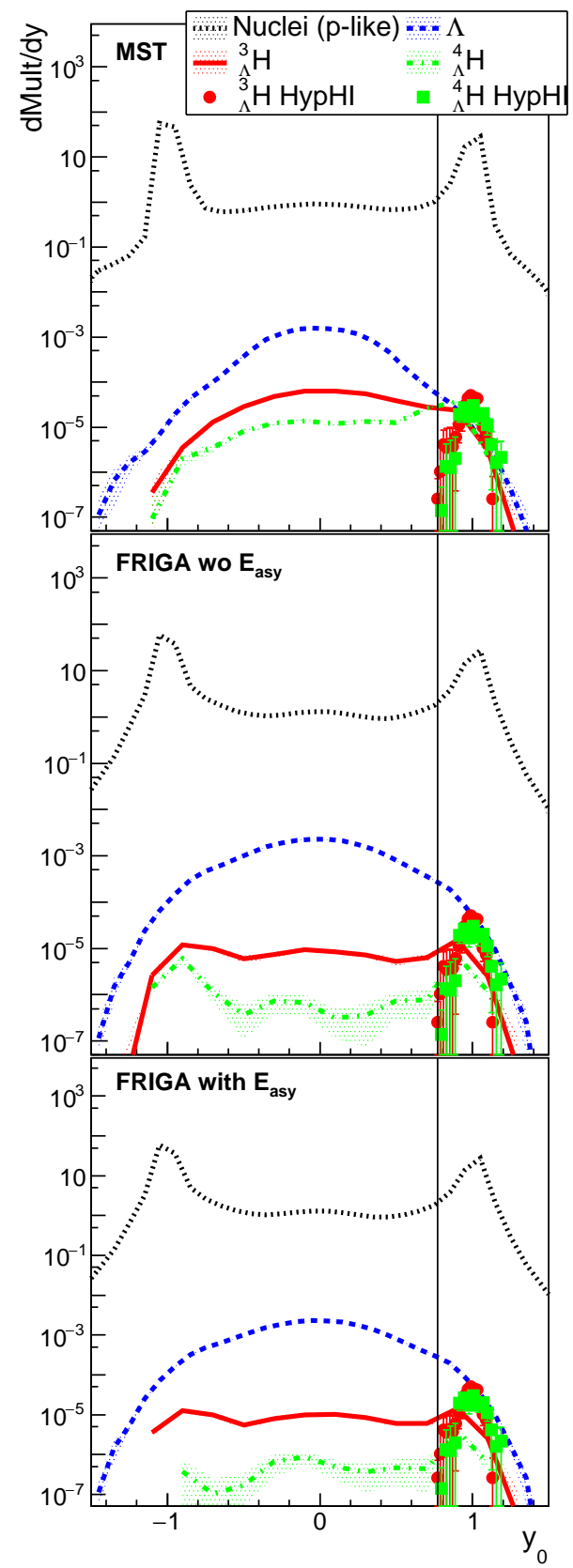

FIG. 12. FRIGA predictions from IQMD (SM EoS) calculations of ${ }^{6} \mathrm{Li}+{ }^{12} \mathrm{C}$ collisions at $2 \mathrm{AGeV}$ incident energy for an impact parameter $2.0<b<5.5 \mathrm{fm}$ at twice the passing time: rapidity dependance of yields per event per unit of rapidity of $\Lambda_{0}$ hyperons and clusters, compared with HypHI experimental yields of hyper-tritons ${ }_{\Lambda}^{3} H$ (red dots) and ${ }_{\Lambda}^{4} H$ (green squares) from [36]. Model predictions of yields of overall nuclei (in proton-like weighting), $\Lambda_{0}$ hyperons, hyper-tritons and ${ }_{\Lambda}^{4} H$ are indicated respectively with black dotted, blue dashed, red full and green dashed-dotted lines. The results of the model calculations are not filtered for the experimental acceptance. The rapidity is expressed in the reference frame of the nucleon-nucleon centre of the colliding system, and scaled to the projectile rapidity. The vertical full line indicates the rapidity above which the HypHI acceptance is alleged to be close to $100 \%$. Below, the experimental acceptance limits the available phase space. Top panel: with clustering done with the MST method. Middle panel: with clustering done by FRIGA with only the basic potential. Bottom panel: with clustering done by FRIGA with asymmetry energy in addition (with $\gamma_{a s y}=1$ ).

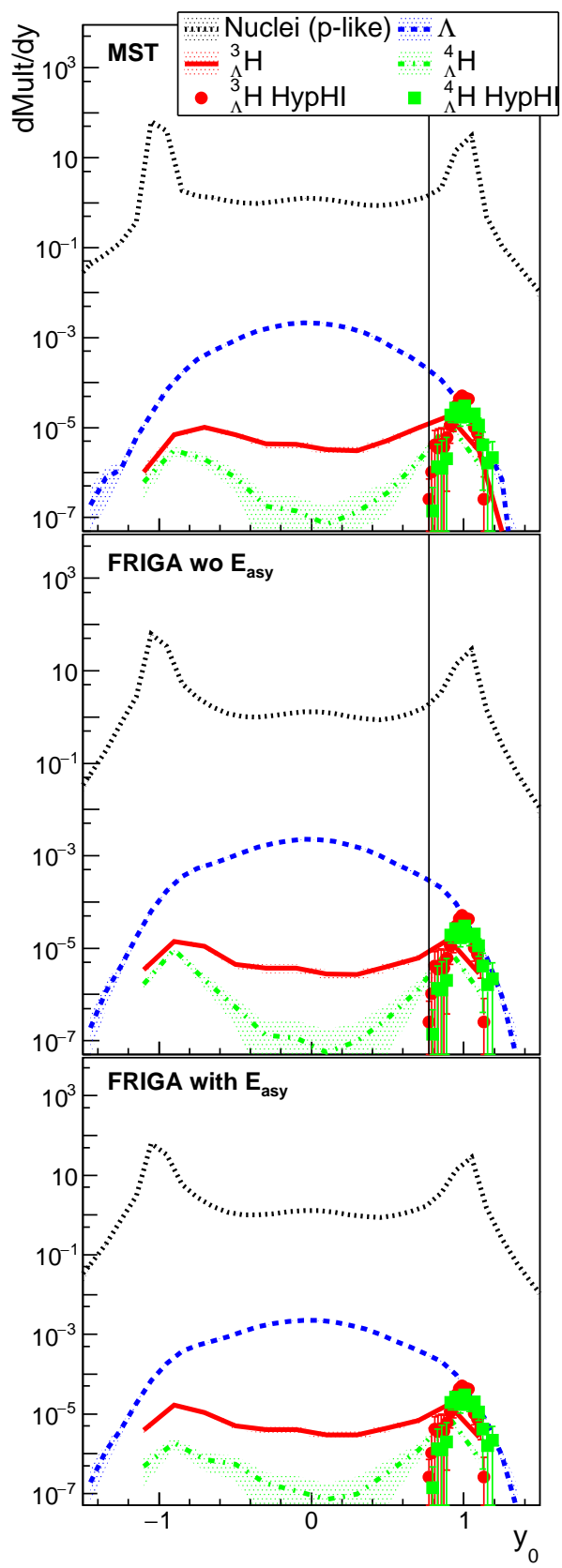

FIG. 13. Same as Fig. 12 with FRIGA clustering performed at four times the passing time.

yields in FRIGA. The same is observed for hyper-nuclei. We have observed that the reduction factor $\frac{2}{3}$ in $V_{\Lambda N}$ has a noticeable effect by decreasing the average hyper-nuclei yields by around 20 percent. The asymmetry energy in the cluster can have a similar effect, depending on the core $(\mathrm{Z}, \mathrm{N})$ asymmetry.

In order to illustrate the predictive power of the FRIGA algorithm, we confront results to experimental observations of light hyper-nuclei produced in the projectile spectator region in collisions of ${ }^{6} \mathrm{Li}+{ }^{12} \mathrm{C}$ at $2 \mathrm{~A}$ 


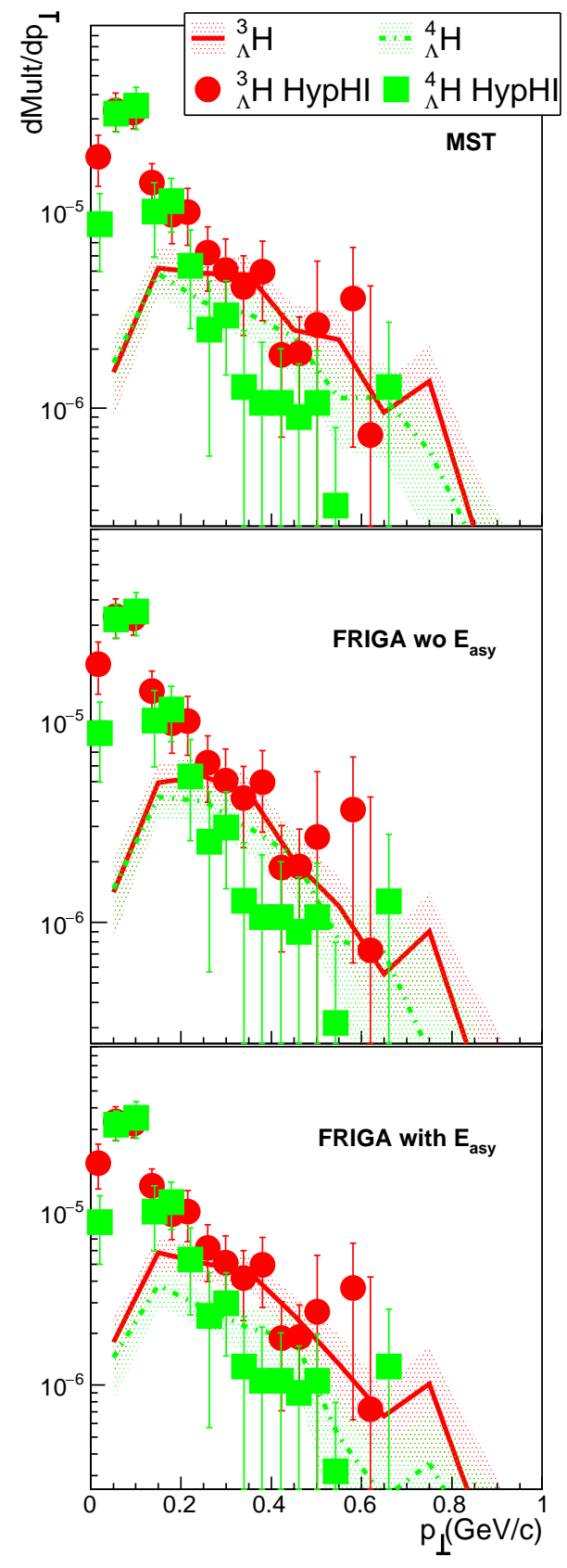

FIG. 14. Same as Fig. 12, at four times the passing time, with only hyper-tritons and ${ }_{\Lambda}^{4} H$ in the representation of the transverse momentum in the projectile spectator region corresponding to the HypHI acceptance. Experimental data are extracted from [36].

GeV incident energy, measured by the HypHI collaboration at the SIS18 synchrotron of GSI Darmstadt. The data are taken from 36]. Fig. 12 compares the IQMDFRIGA predictions with the experimental rapidity distributions of ${ }_{\Lambda}^{3} H$ and ${ }_{\Lambda}^{4} H$. The acceptance of the HypHI set-up allows to reconstruct hyper-nuclei starting from a reduced rapidity, $y_{0}=y / y_{\text {proj }} \approx 0.8$ in the nucleonnucleon centre-of-mass system (y and $y_{\text {proj }}$ are the rapid- ity and projectile rapidity in the chosen reference frame, respectively). This corresponds to $y_{l a b}=1.6$ in the laboratory frame. Therefore, we limit our comparison to this rapidity region, assuming that the complex experimental trigger does not require any extra cuts on the simulation data. However, we observed that a better agreement with the very peaked experimental hyper-hydrogen rapidity distribution is obtained when excluding the most central collisions (taking $b>2 \mathrm{fm}$ ), which indicates that the experimental trigger might have favoured peripheral events. Therefore we adopt this centrality cut for the following. From the proton-like distributions predicted by IQMD, we see that the rapidity region chosen by HypHI exhibits the highest hadronic yield and contains still the tail of the $\Lambda$ distribution. Taking MST as cluster recognition method (Fig. 12 top) results in fairly good agreement of the hyper-hydrogens yields in the projectile spectator region. In this region, we note that the MST yield of hyper-nuclei is high enough to create a visible depletion of remaining free $\Lambda$ hyperons. With the FRIGA approach, we obtain slightly less hyper-hydrogens than with MST. Looking at the yield ratio $Y\left({ }_{\Lambda}^{3} H\right) / Y\left({ }_{\Lambda}^{4} H\right)$ allows to infer the effect of the asymmetry energy of the core nucleus in FRIGA. In the accepted rapidity range, the HypHI experiment has measured a yield ratio $Y\left({ }_{\Lambda}^{3} H\right) / Y\left({ }_{\Lambda}^{4} H\right)=1.4 \pm 0.8$. With the asymmetry energy included in FRIGA, at twice the passing time, the predicted yield ratio is $4.6 \pm 1.1$, whereas without $B_{a s y}$, the ratio goes down to $2.7 \pm 0.5$, because the asymmetry energy tends to reduce the production of ${ }_{\Lambda}^{4} H$, whose core is the isospin-asymmetric triton. However, it turns out that these ratios stabilise in time slightly later in the course of the collision.

In order to probe its persistence with time, we have performed the same comparison at $4 t_{\text {pass }}$. The result is shown in Fig. 13. At the later time, the hyper-hydrogen yields decrease by an order of magnitude in the midrapidity region, but they are not strongly modified in the vicinity of the projectile/target spectator rapidity when using FRIGA. The MST results show a reduction of the projectile/target region yields, which become similar to the ones of the FRIGA approach. The reason is a cooling down of the spectator phase-space. However, the yield ratios $Y\left({ }_{\Lambda}^{3} H\right) / Y\left({ }_{\Lambda}^{4} H\right)$ predicted by FRIGA tend to get smaller, to values which come closer to the experimental results: $2.0 \pm 0.4$ and $2.5 \pm 0.5$ respectively without and with $B_{a s y}$ in the binding energy. Therefore, though the FRIGA parametrisation without $B_{a s y}$ seems to be favoured in comparison with the experiment, the alternative strategy, including asymmetry energy in the core nucleus cannot be ruled-out.

Fig. 14 shows that the distributions of the transverse momentum $p_{\perp}$ in the projectile spectator region agree well (here at four times the passing time), in the slopes and the absolute yields at large transverse momenta with HypHI results, independent of the clustering strategies. A noticeable discrepancy appears at low transverse momenta where the predicted yields are cut-off. This is 
mainly induced by too few low transverse momentum $\Lambda_{0}^{\prime} s$ generated in the spectator region by our present transport model (IQMD). This depletion at low transverse momenta remains unchanged at earlier times. It could explain the yield underestimation that we noticed on the rapidity projection (Figs. 12 and 13).

\section{CONCLUSION}

We present here the first step towards an understanding of the production of isotopic yields and hyper-nuclei in heavy ion reactions. In order to study these we have developed the clusterization algorithm FRIGA, which is based on the SACA approach. The new features include asymmetry and pairing energies as well as shell effects. These are necessary to describe more precisely the nuclear binding energy than it was possible in SACA. The density, temperature and density dependence of these contributions to the binding energy are only vaguely known. They have to be adjusted by comparing the results of the FRIGA algorithm with the existing experimental data. For the interaction between $\Lambda$ and non-strange nucleons we use here a very simplified approach assuming that the strange quarks does not contribute to the interaction. We observe that the asym- metry potential can have a strong influence on the yields of (hyper-)isotopes. According to this model, the nucleons which form fragments have initially a density close to that of their ground state - below the saturation density -, which may differ from the density of the surrounding medium. They contract a little and form finally slightly excited fragments which may undergo secondary decays. Therefore, the fragment formation is sensitive to the subsaturation density dependence of the asymmetry energy. Shell structure effects in primary clusters seem, however, to be of less importance when we compare to the isotope yield.

In this first study we investigated the influence of these new ingredients on the fragment yield and show that the approach allows for realistic predictions of the absolute (hyper-)isotope yield at relativistic energies in the domain of spectator fragmentation as well as of multifragmentation at intermediate energies. In particular, for the first time, HypHI experimental yields of light hypernuclei could be quantitatively predicted within the experimental acceptance.

Acknowledgment : We thank Prof. Elena Bratkovskaya and V. Kireyeu for stimulating discussions and the ALADiN and INDRA collaborations for the permission to use their data prior to publication.
[1] A. Schüttauf et al., Nucl. Phys. A 607 (1996) 457.

[2] W. Reisdorf et al., Nucl. Phys. A 848 (2010) 366-427.

[3] O. Buss et al., Phys. Rept. 512, 1 (2012) doi:10.1016/j.physrep.2011.12.001 arXiv:1106.1344 [hep-ph]].

[4] J. Weil et al., Phys. Rev. C 94, no. 5, 054905 (2016) doi:10.1103/PhysRevC.94.054905 arXiv:1606.06642 [nucl-th]].

[5] C Kuhrts, M Beyer, P Danielewicz, G RÃ $q$ pke, Phys. Rev. C 63 (2001) 034605

[6] H. Feldmeier. Nucl. Phys. A 515 (1990) 147

[7] Ono, Horiuchi et al., Prog. Theor. Phys. 87 (1992) 1185.

[8] W. Bauer, G. F. Bertsch, and S. Das Gupta, Phys. Rev. Lett. 58 (1987) 863

[9] A. Guarnera et al., Phys. Lett. B 373 (1996) 267-274

[10] M. Colonna et al., Nucl. Phys. A 642 (1998) 449-460

[11] J. Aichelin. Phys. Reports 202, 233 (1991).

[12] Ch. Hartnack et al., Eur. Phys. J. A 1 (1998) 151.

[13] S. A. Bass et al., Prog. Part. Nucl. Phys. 41, 255 (1998) [Prog. Part. Nucl. Phys. 41, 225 (1998)]

[14] P. B. Gossiaux, D. Keane, S. Wang and J. Aichelin, Phys. Rev. C 51, 3357 (1995).

[15] J. Steinheimer, K. Gudima, A. Botvina, I. Mishustin, M. Bleicher and H. Stocker, Phys. Lett. B 714, 85 (2012) doi:10.1016/j.physletb.2012.06.069 arXiv:1203.2547 [nucl-th]].

[16] A. S. Botvina, M. Bleicher, J. Pochodzalla and J. Steinheimer, Eur. Phys. J. A 52, no. 8, 242 (2016). doi:10.1140/epja/i2016-16242-7

[17] H.H. Gutrod et al., Phys. Rev. Lett. 37 (1976) 667.

[18] J. Gosset et al., Phys. Rev. C 16 (1977) 629.
[19] M.C. Lemaire et al., Phys. Lett. 85B (1979) 38.

[20] H. Sato and K. Yazaki, Phys. Lett. 98B,3 (1981).

[21] P.B. Gossiaux, R. Puri, Ch. Hartnack, J. Aichelin, Nucl. Phys. A 619 (1997) 379-390.

[22] C. O. Dorso and J. Randrup, Phys. Lett. B 301, 328 (1993).

[23] R. K. Puri and J. Aichelin, J. Comput. Phys. 162, 245 (2000).

[24] K. Zbiri, A. Le Fèvre, J. Aichelin et al., Phys. Rev. C 75 (2007) 034612.

[25] A. Le Fèvre et al., Phys. Rev. C 80 (2009) 044615.

[26] R.J. Charity, GEMINI: A Code to Simulate the Decay of a Compound Nucleus by a Series of Binary Decays, in Joint ICTP-AIEA Advanced Workshop on Model Codes for Spallation Reactions, Report INDC(NDC)0530 (IAEA, Vienna, 2008), p. 139. R. J. Charity, Phys. Rev. C 82 (2010) 014610. D. Mancusi, R. J. Charity, J. Cugnon, Phys. Rev. C 82 (2010) 044610.

[27] K. Zbiri et al., Phys. Rev. C 75, 034612 (2007) doi:10.1103/PhysRevC.75.034612 nucl-th/0607012.

[28] M. Begemann-Blaich et al., Phys. Rev. C 48 (1993) 610.

[29] Sfienti et al., Phys. Rev. Lett. 102 (2009) 152701.

[30] Sfienti et al., Nucl. Phys. A 749 (2005) 83-92.

[31] W. Cassing, E.L. Bratkovskaya, Nucl. Phys. A 831 (2009) 2.

[32] C. Hartnack, H. Oeschler, Y. Leifels, E. L. Bratkovskaya and J. Aichelin, Phys. Rept. 510 (2012) 119

[33] E. Khan, Nguyen Van Giai, N. Sandulescu, Nucl. Phys. A 789 (2007) 94.

[34] A. Le Fèvre et al., Nucl. Phys. A 735 (2005) 219-217.

[35] C. Hartnack, H. Oeschler, Y. Leifels, E. L. Bratkovskaya 
and J. Aichelin, Phys. Rept. $510 \quad$ (2012) 119

arXiv:1106.2083 [nucl-th]].

[36] Ch. Rappold et al., Phys. Lett. B 747 (2015) 129. 